\title{
THE ANALYSIS OF BURROWS RECOGNITION ACCURACY IN XINJIANG'S PASTURE AREA BASED ON UAV VISIBLE IMAGES WITH DIFFERENT SPATIAL RESOLUTION
}

\author{
D. Sun ${ }^{1,2}$, JH. Zheng ${ }^{1,2,3,{ }^{*}}, \mathrm{~T}_{\mathrm{M}}{ }^{1,2}, \mathrm{JJ}^{\mathrm{C}} \mathrm{Chen}^{4}, \mathrm{X}^{\mathrm{L}} \mathrm{Li}^{4}$ \\ ${ }^{1}$ College of Resource and Environment Science, Xinjiang University, Shengli Road 666, Urumchi, China - yukipine@ 163.com \\ ${ }^{2}$ Key Laboratory of Oasis Ecology Ministry of Education, Shengli Road 666, Urumchi, China - zheng_jianghua@ 126.com \\ ${ }_{3}^{3}$ Institute of Arid Ecology and Environment Xinjiang University, Shengli Road 666, Urumchi, China -zheng_jianghua@126.com \\ ${ }^{4}$ Xinjiang Uygur Autonomous Region locust \& rodent forecast \& control Center, Xinhua South Road 23, Urumchi, China -
} 1009669647@qq.com

KEY WORDS: Rodent disaster monitoring, UAV, Low altitude remote sensing, Multi-spatial scale, Object-based image analysis, Grassland protection

\begin{abstract}
:
The rodent disaster is one of the main biological disasters in grassland in northern Xinjiang. The eating and digging behaviors will cause the destruction of ground vegetation, which seriously affected the development of animal husbandry and grassland ecological security. UAV low altitude remote sensing, as an emerging technique with high spatial resolution, can effectively recognize the burrows. However, how to select the appropriate spatial resolution to monitor the calamity of the rodent disaster is the first problem we need to pay attention to. The purpose of this study is to explore the optimal spatial scale on identification of the burrows by evaluating the impact of different spatial resolution for the burrows identification accuracy. In this study, we shoot burrows from different flight heights to obtain visible images of different spatial resolution. Then an object-oriented method is used to identify the caves, and we also evaluate the accuracy of the classification. We found that the highest classification accuracy of holes, the average has reached more than $80 \%$. At the altitude of $24 \mathrm{~m}$ and the spatial resolution of $1 \mathrm{~cm}$, the accuracy of the classification is the highest We have created a unique and effective way to identify burrows by using UAVs visible images. We draw the following conclusion: the best spatial resolution of burrows recognition is $1 \mathrm{~cm}$ using DJI PHANTOM-3 UAV, and the improvement of spatial resolution does not necessarily lead to the improvement of classification accuracy. This study lays the foundation for future research and can be extended to similar studies elsewhere.
\end{abstract}

\section{INTRODUCTION}

Xinjiang is one of the important grassland animal husbandry bases in China. It has a total area of $5,725,800$ hectares of natural grassland, of which 4,860,800 hectares can be used, ranking third in the country (Yan DJ., 2015). Grassland rodent pests refer to rodents that inhabit grasslands have exceeded the environmental carrying capacity due to their high population density, which has adversely affected grassland health and animal husbandry development (Yang YP., 2016). The Yellow Steppe Lemming (Eolagurus luteus) is mainly distributed in northern areas in Xinjiang, and the rodent pests in Ba Yin Gou pasture in $\mathrm{Wu} \mathrm{Su} \mathrm{City} \mathrm{is} \mathrm{the} \mathrm{most} \mathrm{typical.} \mathrm{Not} \mathrm{only} \mathrm{do} \mathrm{these}$ rodents eat a large amount of the stems and leaves of plants, but also destroy their roots and seeds, seriously endangering the growth of grasses. At the same time, the rodent excavation behavior changed the physical and chemical properties of the surface soil, resulting in deep calcium deposits accumulated in the hole. This not only affects the growth of pasture, but also is vulnerable to eolian erosion, eventually causing a decrease in forage grass cover and exacerbating the desertification process in the steppe.

The traditional methods for rodent pest detection mainly include laying patterns, laying traps, and artificial observations (Sheng Z.H., 2015; Hajjaran H., 2013). Ma Yong studied the distribution and ecological habits of the Yellow Steppe Lemming in the Mu Lei County of northern Xinjiang through nearly four months of continuous field observations (Ma Y., 1982). Although this survey has yielded convincing survey results, such survey methods still require a lot of manpower and material costs, and there are also issues such as lack of timeliness and accuracy, which cannot satisfy the demand for researchers to obtain the real-time and accurate disaster information. With the development of remote sensing technology, the monitoring of rodent disaster has entered a new stage. Scholars at home and abroad used satellite images such as Landsat-8 (Li, PX., 2016), Quick Bird (Addink E.A., 2010) and SPOT-5 (Wilschut L.I., 2013) to monitor rat damage, but not as drones could obtain higher spatial resolution images.

As a new type of remote sensing method, low altitude remote sensing of drones has been widely used in landscape ecology and ecology field in recent years because of its high space, time resolution, flexible operation, and low cost (Zhang ZM., 2017). Nowadays, the use of drones for rodent disaster monitoring has only just begun. Ma Tao studied the coverage and distribution characteristics of Rhombomys opimus in the Gurbantunggut desert forest based on UAV images (Ma T., 2018). However, these studies have failed to compare the accuracy of burrows recognition for aerial images with different spatial resolution scales.

Based on this, this paper selected $\mathrm{Ba}$ Yin Gou ranch in $\mathrm{Wu} \mathrm{Su}$ City as a research area, and used consumer-grade DJI PHANTOM-3 as low-altitude remote sensing platforms to

\footnotetext{
${ }^{1}$ Corresponding Author: Email: zheng_jianghua@ 126.com
} 
collect images with different flying heights. In this paper, the photos were categorized and the spatial resolution of images at different altitudes was calculated. Then, based on the images of these different spatial resolutions, the burrows were identified and extracted, and the recognition accuracy was compared, which has important guiding significance for the later monitoring of rodent disasters.

\section{RESEARCH AREA}

The experimental area (Fig. 1) is located in Ba Yin Gou Pasture $\left(84^{\circ} 59^{\prime} 55^{\prime \prime} \mathrm{E} 44^{\circ} 12^{\prime} 56^{\prime \prime} \mathrm{N}\right)$ in Wu Su City, Xinjiang, China. It is located in the semi-desert grassland, where the vegetation types are mainly Seriphidium transiliense, Anabasis aphylla, Salsolacollina Pall and other low-vegetation plants. Typical rodent disaster in the experimental area is Yellow Stepped Lemming (Eolagurus luteus).

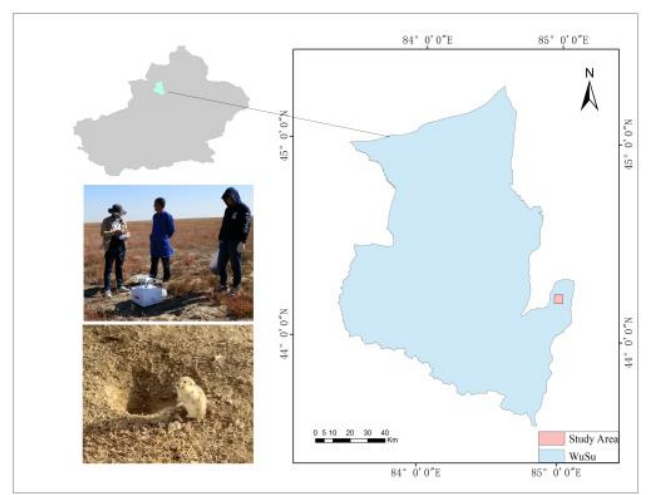

Figure 1.Study Area

\section{DATA AND METHODS}

\subsection{Data Source}

The remote sensing platform we used in this study is the DJI PHANTOM-3 UAV, with the camera type FC300S. The main wavelength range is visible light (RGB), and the detailed parameters are shown in Tab.1. The flight time is September 27, 2017. In this paper, we selected four images with flight heights of 3 meters, 6 meters, 24 meters, and 30 meters for analysis.

\begin{tabular}{cc}
\hline \multicolumn{2}{c}{ UAV platform } \\
\hline Model & DJI PHANTOM-3 \\
Type & $1280 \mathrm{~g}$ \\
Weight & $20 \mathrm{~min}$ \\
Maximum flight time & $57.6 \mathrm{~km} / \mathrm{h}$ \\
Maximum flight speed & $6000 \mathrm{~m}$ \\
Highest flight altitude & \\
\hline & FC 300-S \\
\hline Camera model & $94^{\circ}$ \\
FOV & $3.6 \mathrm{~mm}$ \\
Camera focus & $6.16 \mathrm{~mm} * 4.62 \mathrm{~mm}$ \\
Sensor size & $4000^{*} 3000$ \\
Maximum resolution & 12.4 million \\
Effective pixels &
\end{tabular}

Table 1. UAV and sensor parameters

\subsection{Images Spatial Resolution Calculation}

The calculation of UAV image resolution is an important data pre-processing task. The spatial resolution of an image refers to the size of the actual ground size which the pixels on the image are mapped, and the smaller the size, the higher the resolution (Yang RS., 2013). For UAV systems, the flying height has a great influence on the resolution of the image, and the higher the flying height, the lower the spatial resolution of the image (Fig.2). The spatial resolution of the image is also affected by camera parameters. The image resolution is calculated as follows:

$$
\mathrm{GSD}=(\mathrm{H} \times \alpha) / f
$$

Where $\mathrm{GSD}=$ ground resolution in units of $\mathrm{m}$ $H=$ flight height in $\mathrm{m}$ $\alpha=$ pixel size in $\mu \mathrm{m}$ $f=$ camera focal length in $\mathrm{mm}$

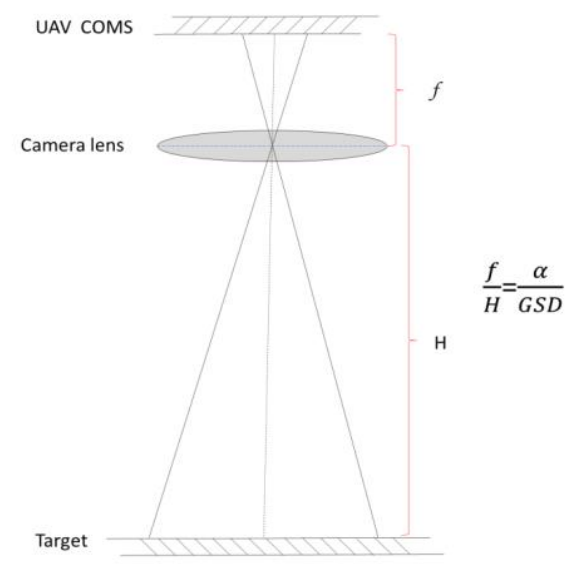

Figure 2.Image Spatial Resolution Calculation

\subsection{Object-oriented Burrows Extraction}

The principle of object oriented classification (Peña J.M., 2013) is to first segment images into many objects, each of which is homogeneous, and there are differences between objects and objects. Classification is based on objects, not based on a single pixel, which can greatly improve the classification accuracy and reduce the classification noise. For image segmentation, this paper is carried out under the eCognition software.

In this paper, a common multi-resolution segmentation algorithm (Rahman M.R., 2008) is used to merge images from bottom to top into many image objects based on the principle of internal homogeneity. Segmentation parameters mainly include scale, colour, shape, smoothness, and compactness. The smaller the parameter is set, the more the number of objects after the segmentation is, and the more broken the segmentation is. The larger the parameter setting, the smaller the number of objects after the segmentation, and the greater the possibility that the feature will be misclassified. In this paper, we set up different segmentation parameters (Table 2) through many experiments. By comparing the effect of segmentation (Figure 3), we finally determined the best segmentation parameters of four images.

\begin{tabular}{ccccc}
\hline A & Level 1 & Level 2 & Level 3 & Level 4 \\
\hline Scale & 30 & 60 & 100 & 150 \\
Shape & 0.5 & 0.5 & 0.5 & 0.5 \\
Color & 0.5 & 0.5 & 0.5 & 0.5 \\
Compact & 0.8 & 0.8 & 0.8 & 0.8 \\
Smooth & 0.2 & 0.2 & 0.2 & 0.2 \\
Object & 32913 & 7882 & 2570 & 1008 \\
number & & & & \\
\hline B & Level 1 & Level 2 & Level 3 & Level 4 \\
Scale & 20 & 30 & 50 & 100
\end{tabular}




\begin{tabular}{ccccc} 
Shape & 0.5 & 0.5 & 0.5 & 0.5 \\
Color & 0.5 & 0.5 & 0.5 & 0.5 \\
Compact & 0.8 & 0.8 & 0.8 & 0.8 \\
Smooth & 0.2 & 0.2 & 0.2 & 0.2 \\
Object & 53371 & 29054 & 10151 & 3685 \\
number & & & & \\
\hline C & Level 1 & Level 2 & Level 3 & Level 4 \\
\hline Scale & 20 & 20 & 30 & 50 \\
Shape & 0.2 & 0.5 & 0.5 & 0.5 \\
Color & 0.8 & 0.5 & 0.5 & 0.5 \\
Compact & 0.5 & 0.8 & 0.5 & 0.5 \\
Smooth & 0.5 & 0.2 & 0.5 & 0.5 \\
Object & 68512 & 48025 & 23851 & 7802 \\
number & & & & \\
\hline D & Level 1 & Level 2 & Level 3 & Level 4 \\
\hline Scale & 20 & 20 & 30 & 50 \\
Shape & 0.2 & 0.5 & 0.5 & 0.5 \\
Color & 0.8 & 0.5 & 0.5 & 0.5 \\
Compact & 0.5 & 0.8 & 0.5 & 0.5 \\
Smooth & 0.5 & 0.2 & 0.5 & 0.5 \\
Object & 67183 & 44591 & 25234 & 8603 \\
number & & &
\end{tabular}

Table 2. Multi-resolution segmentation parameters

Spectral difference segmentation strictly speaking cannot be regarded as a segmentation algorithm, it cannot create a new segmentation layer based on the pixel layer, but based on the existing segmentation layer, by analysing whether the brightness difference of the adjacent segmentation objects meets the given threshold to decide when to merge objects. Multiresolution segmentation combined with spectral difference segmentation can merge objects with relatively similar brightness values, reduce the number of segmentation objects, and optimize the segmentation results.

Supervised classification, also known as training classification, refers to the technique of selecting training samples for classification through prior knowledge. The traditional supervised classification is based on pixel-level classification, while the supervised classification of the object-oriented method is based on the segmented objects. Object-based supervised classification can reduce the noise generated by image classification, resulting in more accurate classification results.

\section{RESULTS AND ANALYSIS}

\subsection{Images spatial resolution calculation}

The FC $300-\mathrm{S}$ sensor size is $1 / 2.3$ inch and the pixel size is 1.5 $\mu \mathrm{m}$. According to formula (1) we calculated the spatial resolution of the four images as Table 3 . In image A, we can clearly see the burrows. And the width of the burrows is about 50-60 pixels. While in image $\mathrm{D}$, the burrows is only about 4-6 pixels wide.
Figure 3. Different spatial resolution images

\begin{tabular}{ccccc}
\hline Image & $\mathrm{A}$ & $\mathrm{B}$ & $\mathrm{C}$ & $\mathrm{D}$ \\
\hline $\mathrm{H}$ & $3 \mathrm{~m}$ & $6 \mathrm{~m}$ & $24 \mathrm{~m}$ & $30 \mathrm{~m}$ \\
$\mathrm{GSD}$ & $0.125 \mathrm{~cm}$ & $0.25 \mathrm{~cm}$ & $1 \mathrm{~cm}$ & $1.25 \mathrm{~cm}$ \\
\hline
\end{tabular}

Table 3. GSD calculation for four images

\subsection{Images segmentation results}

Comparing the multi-resolution segmentation results of local regions, we found that the Level 1 segmentation scale was insufficient and the map spot is too fragmented on the A image. In the Level 2 segmentation scale, although the burrows segmentation effect was ideal, the large bare land and the grassland were fragmented too much. At the Level 4 scale, burrows were not well segmented. Therefore, Level 3 was selected as the optimal segmentation layer (the effect of mouse hole segmentation can be seen in the red range). Similarly, compared with the other three images, the optimal segmentation layers are: B: Level 3, C: Level 3, and D: Level 2.

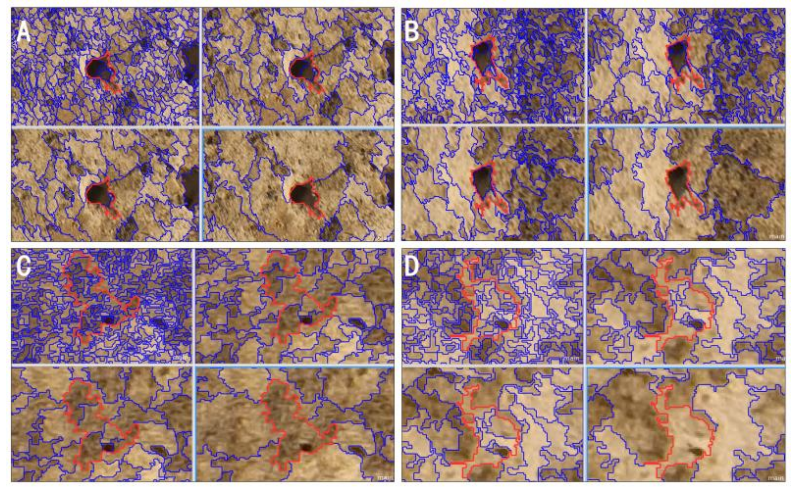

Figure 4. Multi-resolution segmentation results

\subsection{Burrow extraction and accuracy assessment.}

According to the classification results of UAV images, we found that burrows were clearly visible on aerial images. From the results of the extraction, there are some errors in the burrows classification results. The dark shades of some vegetation and the clefts on the ground became clearer, which lead that the computer interpreting has a wrong identification. At the same time, some of the bare land samples were classified into grasslands. This may be due to the aerial time of autumn, when the grass was withered and yellow, and there were traces of disturbance in the bare land. These caused the bare reflectance spectrum characteristics to be very similar to that of the yellow forage.

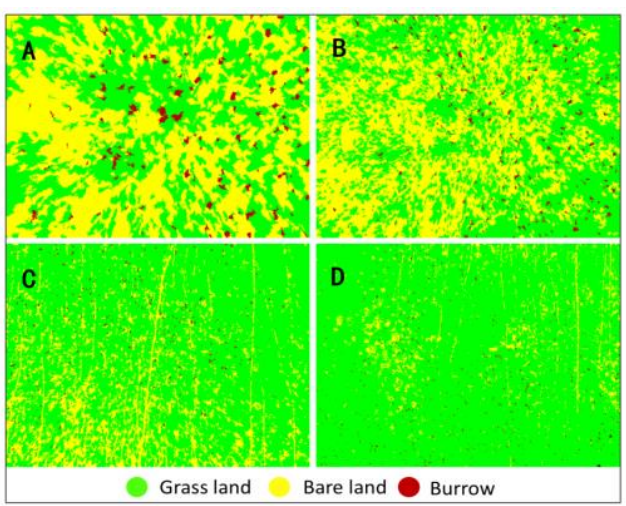


Figure 5. Burrow classification results

\begin{tabular}{|c|c|c|c|c|}
\hline A & & Burrow & \multicolumn{2}{|c|}{ Grassland } \\
\hline Burrow & & 15 & \multicolumn{2}{|c|}{11} \\
\hline Grassland & & 0 & \multicolumn{2}{|c|}{79} \\
\hline Bare-land & & 0 & \multicolumn{2}{|c|}{10} \\
\hline Sum & & 15 & \multicolumn{2}{|c|}{100} \\
\hline B & & Burrow & \multicolumn{2}{|c|}{ Grassland } \\
\hline Burrow & & 25 & \multicolumn{2}{|c|}{6} \\
\hline Grassland & & 5 & \multicolumn{2}{|c|}{80} \\
\hline Bare-land & & 0 & \multicolumn{2}{|c|}{14} \\
\hline Sum & & 30 & \multicolumn{2}{|c|}{100} \\
\hline $\mathrm{C}$ & & Burrow & \multicolumn{2}{|c|}{ Grassland } \\
\hline Burrow & & 52 & \multicolumn{2}{|c|}{7} \\
\hline Grassland & & 6 & \multicolumn{2}{|c|}{187} \\
\hline Bare-land & & 2 & \multicolumn{2}{|c|}{6} \\
\hline Sum & & 60 & \multicolumn{2}{|c|}{200} \\
\hline $\mathrm{D}$ & & Burrow & \multicolumn{2}{|c|}{ Grassland } \\
\hline Burrow & & 84 & \multicolumn{2}{|c|}{19} \\
\hline Grassland & & 16 & \multicolumn{2}{|c|}{180} \\
\hline Bare-land & & 0 & \multicolumn{2}{|c|}{1} \\
\hline Sum & & 100 & \multicolumn{2}{|c|}{200} \\
\hline \multicolumn{5}{|c|}{ Table 4. Sample confusion matrix } \\
\hline Image & A & $\mathrm{B}$ & $\mathrm{C}$ & $\mathrm{D}$ \\
\hline $\begin{array}{l}\text { Overall } \\
\text { accuracy }\end{array}$ & $75.3 \%$ & $78.7 \%$ & $92.8 \%$ & $85.8 \%$ \\
\hline KIA & 0.578 & 0.649 & 0.881 & 0.778 \\
\hline
\end{tabular}

Table 5. Classification accuracy verification

In order to evaluate the accuracy of the classification results, we randomly selected a certain number of samples on the four images by visual interpretation and evaluated the classification results. According to the accuracy evaluation confusion matrix (Tab.4\&5), we found that:

(1) Burrow samples were well classified when the flying height was $3 \mathrm{~m}$ (GSD, $0.125 \mathrm{~cm}) .11 \%$ of grassland samples were classified as burrows because grass shadows and burrows are more difficult to distinguish. While, there were $31 \%$ of bare samples have been classified as grassland.

(2) When the flying height of $6 \mathrm{~m}(\mathrm{GSD}, 0.25 \mathrm{~cm})$, the classification accuracy of burrow reached $83.3 \%$, while some grassland samples were misclassified into burrow and bare land, and $24 \%$ of bare land samples were misclassified into grassland.

(3) When the flying height is of $24 \mathrm{~m}$ (GSD, $1 \mathrm{~cm}$ ), the classification accuracy of burrow reached $86.7 \%$, and the classification accuracy of grassland and bare land has increased to more than $90 \%$, which lead the improvement of the overall classification accuracy $(92.8 \%)$.

(4) When the flying height is of $30 \mathrm{~m}(\mathrm{GSD}, 1.25 \mathrm{~cm})$, the classification accuracy of the burrow was $84 \%$, the grassland classification accuracy was $90 \%$, and the bare land classification accuracy was $82.5 \%$.

From Tab.5, we found that the classification accuracy of burrow is the best, with an average of more than $80 \%$. With the reduction of spatial resolution, the classification accuracy of grassland has also been improved, and the discrimination between bare land and grassland has become more pronounced. At a flying height of $24 \mathrm{~m}$ and a spatial resolution of $1 \mathrm{~cm}$, the classification accuracy of the object is the highest (Overall Accuracy $92.8 \%$, KIA 0.88).

\section{CONCLUSIONS AND DISCUSSION}

In this paper, $\mathrm{Ba}$ Yin Gou pasture was selected as research area and DJI PHANTOM-3 as remote sensing platform. We used four different flying heights $(3 \mathrm{~m}, 6 \mathrm{~m}, 24 \mathrm{~m}$, and $30 \mathrm{~m})$ to shoot the rat holes and calculated the spatial resolution of the images corresponding to the four altitudes. We used the object-oriented supervised classification method to extract the four mouse holes. And based on the random samples, the accuracy of the classification results was evaluated. We the conclusion that when the altitude is $24 \mathrm{~m}$, the image spatial resolution is $1 \mathrm{~cm}$, the extraction accuracy of burrow is the highest.

With the increase of altitude and the decrease of spatial resolution of images, the classification accuracy of grassland and bare land has also been improved, which may be due to the excessive spatial resolution that can amplify the detailed features of the features. This will cause some confusion in the image classification, thus reducing the classification accuracy. Obviously, under certain circumstances, blind pursuit of excessively high spatial resolution may not necessarily lead to an increase in the classification accuracy, but it also inevitably results in a problem of excessive data volume.

The burrows recognition accuracy is about $80 \%$, it because that the spectral reflection characteristics of dark vegetation shadow and the rat hole are relatively close. In the future, the spectral characteristics features will be considered to improve the rat hole recognition accuracy.

\section{ACKNOWLEDGEMENTS}

Supported by Xinjiang uygur autonomous region youth science and technology innovation talent training project (2016); Grassland biological disaster monitoring in Xinjiang based on remote sensing (2017). We sincerely thank the Xinjiang Uygur Autonomous Region Production Education Research Joint Training Base of Postgraduate that provides a great support for this study.

\section{REFERENCES}

Addink E.A., Jong S.M.D., Davis S.A., 2010. The use of high-r esolution remote sensing for plague surveillance in Kazakhstan [J]. Remote Sensing of Environment, 114(3), pp. 674-681, https: //doi.org/10.1016/j.rse.2009.11.015

Hajjaran H., Mohebali M., Abaei M.R., 2013. Natural infection and phylogenetic classification of Leis-hmania spp. infecting $\mathrm{Rh}$ ombomys opimus, a primary reservoir host of zoonotic cutaneo us leishmani-asis in northeast Iran[J]. Transactions of the Royal Society of Tropical Medicine and Hygiene, 107(9), pp. 550-557, doi: $10.1093 /$ trstmh/trt060

Li, PX., Zheng JH., Ni YF., 2016. Estimating Area of Grassland Rodent Damage Rangeland and Rat Wastelands Based on Rem ote Sensing in Altun Mountain, Xinjiang, China[J]. Xinjiang A gricultural Sciences, 53(07), pp. 1346-1355,doi: 10.6048/j.issn. 1001-4330.2016.07.023 
Ma Y., Wang F.G., Jin S.K., 1982. On distribution and ecology of yellow steppe lemming (lagurus luteus ) of Xinjiang [J]. Act a Theriologica Sinica, 1982(01), pp. 81-88.

Ma T., Zheng JH., Wen AM., 2018. Group coverage of burrow entrances and distribution characteristics of desert forest- dwell ing Rhombomys opimus based on unmanned aerial vehicle (UA V) low- altitude remote sensing: A case study at the southern margin of the Gurbantunggut Desert in Xinjiang[J]. Acta Ecolo gica Sinica, 38(3), pp. 953-963, doi: 10.5846 /stxb2016121425 71

Peña J.M., Torressánchez J., de Castro A.I., 2013. Weed mappi $\mathrm{ng}$ in early-season maize fields using object-based analysis of $\mathrm{u}$ nmanned aerial vehicle (UAV) images[J]. Plos One, 8(10):e771 51,doi: 10.1371/journal.pone.0077151

Rahman M.R., Saha S.K., 2008. Multi-resolution segmentation for object-based classification and accuracy assessment of land use/land cover classification using remotely sensed data[J]. Jour nal of the Indian Society of Remote Sensing, 36(2), pp. 189-201, https://doi.org/10.1007/s12524-008-0020-4

Sheng Z.H., Chen M., Liu Z.J., 2015. Importance of loss evalua tion indexes of rodent disaster in the desert forests in Changji,X injiang Uygur Autonomous Region[J]. Forest Pest \& Disease, 34 (01),pp. 14-17,22,doi:10.3969/j.issn.1671- 0886.2015.01.004

Wilschut L.I., Addink E.A., Heesterbeek J.A., 2013. Mapping t he distribution of the main host for plague in a complex landsca pe in Kazakhstan: An object-based approach using SPOT-5 XS, Landsat 7 ETM+, SRTM and multiple Random Forests[J]. Int J Appl EarthObs Geoinf, 23(100), pp. 81-94, https://doi.org/10.1 016/j.jag.2012.11.007

Yan DJ., Zhou QW., Hao LU., 2015. The Disaster, Ecological Distribution and Control of Poisonous Weeds in Natural Grassl ands of Xinjiang Uygur Autonomous Region[J]. Scientia Agric ultura Sinica,48(03),pp. 565-582,doi: 10.3864/j.issn.0578-1752. 2015.03.16

Yang YP., Zhang FS., Wang LQ., 2016. Comprehensive preven tion and control technology of grassland rodent[M].Chinese agr icultural science and Technology Press.

Yang RS., Ma YY., Yin HZ., 2013. Relationship between Grou nd Resolution and Flying Height about the Low Altitude Unma nned Aerial Vehicle Aerophotographic System.[J]. Surveying a nd Mapping of Geology and Mineral Resources, (3), pp. 1-2, 8, doi: 10.3969/j.issn.1007-9394.2013.03.001

Zhang ZM., X Q., Wang B., 2017. Applications of unmanned a erial vehicles remote sensing technology in landscape ecology [J]. Acta Ecologica Sinica, 37(12), pp. 4029-4036,doi: 10.5846/ stxb201702270318 